betteraves, son, farine d'orge ; peu à peu, on diminua la quantité de lait, jusqu'à la suppression définitive ; aussitôt a près le sevrage, les animaux restèrent à la prairie. Aussi bien pour l'une que pour l'autre race, GrRARD et BARDin constatèrent que la croissance était en rapport direct a vec le poids à la naissance.

L'augmentation moyenne journalière établie sur une période variant de 5 à 7 mois fut :

pour les Southdown, 177 gr. 50 ;

pour les Berrichons, $191 \mathrm{gr}$.

A l'âge où l'on peut le sevrer, c'est-à-dire à 3 mois, un agneau pèse 22 kilogs ; il a done augmenté son poids de naissance de 5,5 fois environ.

En 30 jours, le poids de naissance du chevreau a fait plus que doubler; l'augmentation totale est de 5 kilogs en chiffres ronds, ce qui fait un accroissement quotidien de 160 grammes environ. La croissance du chevreau est moins rapide que celle de l'agneau : à trois mois, le premier pèse 22 kilogs. Chez l'agneau, le poids de naissance est doublé en 17 jours ( 15 jours d'a près ABDERHALDEN) ; en $22-23$ jours, chez le chevreau.

(A suivre.)

\title{
VARIATIONS QUANTITATIVES ET QUALITATIVES DE LA PRODUCTION DU LAIT CHEZ LA VACHE SOUS L'INFLUENCE DE LA CASTRATION (1)
}

par

\section{J. ALQUIER et Mile $G$. SILVESTRE DE SACY}

(Travail du Laboratoire de Recherches et de la Station d'expérimentation sur l'alimentation du bétail, de la Société Scientifique d'Hygiène Alimentaire et de l'Institut des Recherches agronomiques.)

La production du lait dépend, avant tout, de la race, de l'aptitude et des conditions individuelles de l'animal, ainsi que de l'état de la glande mammaire à la période considérée de la lactation. L'alimentation, les influences extérieures et les autres facteurs n'agissent, en général, qu'en seconde ligne. L'étude de l'influence des aliments sur la sécrétion lactée est, par conséquent, des plus ardues.

La glande mammaire atteint son maximum possible de production dans les premiers temps qui suivent le part, et c'est à cette époque que l'action des aliments s'exerce au maximum. Dès que l'activité glandulaire, qui suit la naissance du veau, s'est un peu modérée, l'alimentation, quelle qu'elle soit, quantitativement ou qualitativement, ne saurait entretenir le rendement en lait du début.

(I) Travail paru dans le Bulletin de la Société Scientifique d'Hyg. alim. 1930, p. 369. 
On conçoit dès lors que, si l'on arrivait à maintenir, un certain temps, cette production élevée de lait, qui suit le vêlage, l'étudé de l'influence des aliments sur la lactation se trouverait grandement facilitée. On peut, en effet, pour une race donnée, atténuer, entre autres, l'action si grande de l'individualité, en expérimentant sur un nombre suffisamment élevé de têtes.

Telles sont les raisons qui nous ont laissé entrevoir un certain intérêt aux recherches se proposant d'étudier l'influence de la castration sur la production quantitative et qualitative du lait chez la vache. L'ovariotomie figure, on l'a déclaré maintes fois, parmi les moyens artificiels de prolonger notablement l'activité mammaire du début de la lactation, puis de la régulariser. Or, la baisse continue de la production, avec ses sauts si fréquents au cours de la lactation, est justement la cause principale d'erreur qui interdit, souvent, toute conclusion, Iorsque l'on recherche méthodiquement l'action de l'alimentation sur la production laitière.

Les vétérinaires (1) ont pratiqué couramment, avant 1900, la castration de la vache ; ils se sont trouvés d'accord pour reconnaître que cette opération prolonge la sécrétion pendant 20 ॠ 24 mois, qu'elle régularise la qualité du lait, facilite l'engraissement et améliore la qualité de la viande. Mais ces praticiens ne donnent aucune indication précise, prineipalement sur les variations de la composition chimique du lait, consécutives à la castration et les opinions les plus contradictoires ont été publiées dans la presse agricole à ce sujet.

$$
*^{*} *
$$

M. le Dr Lermat, vétérinaire de la Chambre syndicale des Lai tiers nourrisseurs de Paris, après avoir suivi l'évolution de la question, a pu, il y a quelques années, en présenter la première étude méthodique (2), basée sur son expérience personnelle après une pratique, on peut le dire, de tous les jours.

L'auteur a discuté, de différents points de vue, les résultats économiques de la castration des vaches. Voici ses conclusions.

Tout d'abord, cette opération, pratiquée par un spécialiste, reste à peu près sans danger.

A partir du jour où elle a été privée de ses ovaires, la vache ne revient plus en chasse, surtout si elle est taurelière, continue à donner son lait, retrouve le calme absolu, ne dérange plus les autres bêtes au moment du rut et engraisse facilement.

(1) Entre autres: C. Flocard, médecin vétérinaire, Rapport sur la Castration des vaches laitières, Bulletín $n^{\circ}$ 141, de la classe d'Agriculture de la Société des Arts de Genève (1er tri. mestre 1895). G. Buwas., Les avantages de la Castration (Meaux 1895).

(2) H. Leratat : in «Ingénieur agricole de Gembloux », 1er novembre, 1898. L'ovariotomie des vaches laitières, ses résultats. Thèse Doct.-Vét., Ed. médicales, 7, rue de Valois, 1924. 
En règle générale, la séerétion du lait se trouve très notablement prolongée par la eastration, par suite, entre autres, de la suppression tant du retour périodique des chasses que de l'état de gestation qui arrête la lactation quelques semaines avant le vêlage il ne faut pas l'oublier pour cette étude. - Cette augmentation du rendement varierait toutefois selon la race de l'animal, son individualité, son tempérament, sa nourriture et l'hygiène.

La castration passe pour réussir infiniment mieux avec la race suisse qu'avec les autres : elle prolongerait, chez elle, pendant 20 à 24 moís environ le rendement obtenu au moment du vêlage. On eite des vaches suisses castrées, donnant, au cours de l'année qui suit l'opération, de 1.300 à 1.400 litres de plus, toutes choses égales (1), que si elles n'avaient pas été privées de leurs ovaires. Chez les normandes, bêtes plus nerveuses et à tempérament capricieux, moins rustiques en stabulation et dont la durée de lactation est très courte puisqu'elle ne se maintient que de 8 à 10 mois au maxımum, on aurait parfois prolongé l'activité mammaire pendant 18 mois environ. Chez les races du Nord, Hollandaises ou Flamandes, dont les ehasses passent souvent inaperȩues, on aurait également tiré quelques avantages de la castration, mais il ne faut pas l'oublier, les Hollandaises ont un rendement de lait rémunérateur jusqu'à la fin de la troisième année. Quant aux autres races, elles réagiraient de même favorablement à la castration, et l'on a publié à ce sujet des chiffres qui paraissaient convaincants.

La presse italienne enregistre nombre de faits venant à l'appui de l'heureuse influence de la castration (2). En Amérique, plus spécialement en Argentine, en Uruguay, et même au Canada, la castration est une opération de pratique courante, considérée comme intéressante.

En ce qui concerne la qualité du lait, celui-ci acquerrait des propriétés nouvelles après la castration. Le trayeur lui-même sapercevrait que le lait des castrées est plus gras, plus onctueux au toucher. Le consommateur le trouverait plus agréable au goût, plus savoureux et particulièrement plus sucré.

Quant aux analyses publiées de différents côtés, elles ne permettent guère de conclusions très nettes, surtout en ce qui concerne l'augmentation très sensible, affirmée par certains, du beurre et peut-être de la caséine, en tout cas du lactose. On a sans doute cité des analyses de lait de vaches castrées d'une richesse exceptionnelle en matières grasses (plus de $49 \%$ ) et en matières minérales, mais les résultats obtenus de divers côtés permettent-ils de

(1) Ce qui n'est probablement pas exact pour la plupart des cas, car la comparaison a dù être faite avec des bêtes en état de gestation, comme témoins.

(2) Prof. Buoxsaxti : Agricoltura Moderna, no 15, 1898. 
rapprocher, étant donnée sa composition moyenne, le lait des vaches castrées de celui de l'espèce humaine, à le déclarer plus reconstituant grâce à sa minéralisation et à sa richesse en acide phosphorique?

Quant aux effets de la castration au point de vue de l'engraissement, la question n'est mise en discussion par personne. On trouve couramment en Suisse, sur le marché, des bêtes castrées, dites "beuves", marquées à l'oreille pour qu'il n'y ait pas de fraude dans le cas où elles viendraient à être achetées dans tout autre but que la boucherie, et, d'après les observations de l'abattoir de Genève, le rendement en viande nette de ces vaches grasses castrées $(60 \%)$ serait plus élevé de 5 à $6 \%$ que celui des vaches engraissées à l'état de gestation. En Argentine, où l'on a en vue non pais la production du lait, mais l'engraissement de bêtes destinées aux frigorifiques et à la boucherie de qualité, la castration, qui se pratique couramment, occasionnerait un plus grand bénéfice ; les bêtes seraient mieux et plus vite engraissées.

M. LeRMAT, après avoir indiqué dans son mémoire quelles sont les vaches qui ne doivent plus " porter ", conclut que, malgré tous ces résultats favorables, la pratique de l' "ovariotomie" n'est logique que chez les vaches "taurelières " ou "cassées " ou "décrochées ", en un mot chez toutes celles qui sont stériles pour une raison quelconque.

Il recommande enfin d'opérer au printemps, la saison la plus favorable pour que les interventions chirurgicales réussissent, et 2 mois après le vêlage, bien que l'on ait castré des vaches 3 semaines seulement après la naissance du veau.

Telle est, d'après les résultats enregistrés par le praticien le plus compétent en la matière, la mise au point de la question de la castration. Nous avons essayé de la reprendre, pour les raisons précédemment indiquées, en expérimentant plus scientifiquement qu'on

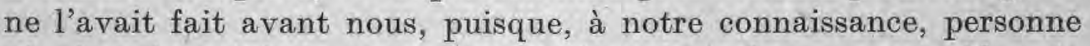
n'a jamais suivi, le temps nécessaire, deux lots comparables de vaches, composés l'un de vaches castrées, l'autre de bêtes non castrées et dont la lactation suivait son cours normal sans être troublée par la gestation.

La méthode dite des "groupes). s'imposait en la circonstance ; nous l'avons précédemment énoncée et discutée dans ce Bulletin (1).

Dans deux lots de vaches, ayant vêlé à peu près à la même époque, à une ou deux semaines près, lots composés, l'un de normandes, l'autre de flamandes, nous avons constitué deux groupes de 4 vaches, comprenant, chacun, 2 normandes et 2 flamandes

(1) J. Alquier : Méthodes d'essais, scientifiques et pratiques, de rationnement du bétail. Bull. de la Soc. Sc. Hyg. Alim. T. xv, 1927, no 6, F. 277 et suiv. 
choisies telles que chaque animal de chaque groupe était comparable à un autre animal de l'autre groupe, comme race, âge, poids vif et rendement.

Les animaux d'expérience ainsi réunis, comme du reste toutes les vaches de l'étable d'expérimentation, ne céssèrent d'être soumis au contrôle laitier, la traite étant pratiquée deux fois par jour au moyen d'une machine déjà décrite (1), qui avait donné toute satisfaction. Le contrôle porta, trois fois par mois environ depuis le vêlage, sur la quantité de lait produite pendant une période de 4 jours consécutifs. L'échantillon du lait moyen de chaque vache, recueilli pendant chacune de ces périodes de 4 jours, était analysé aussi régulièrement que possible.

Le choix des animaux à comparer a duré 3 mois, de novembre à février, sans qu'il ait été possible auparavant d'être certain de l'homogénéité des lots à comparer et d'être assuré que la produetion de chaque groupe était identique à celle de l'autre groupe. Les conditions dans lesquelles les deux groupes étaient placés, telles que l'alimentation, le nombre des repas, les heures de traite, la température de l'étable et les soins donnés, restèrent toutes exactement les mêmes, jusqu'à la fin naturelle de la lactation.

La nécessité où nous nous sommes trouvés de ne faire castrer les bêtes à comparer que 4 mois après leur vêlage, est la seule réserve qu'il soit possible de faire sur ces recherches de longue haleine. On aurait reconnu, en effet, qu'il y avait avantage à pratiquer la castration de la vache dès la fin du deuxième mois après la mise bas. Toutefois, nous le répétons, opérer plus tôt eut enlevé de la valeur à la comparaison des données expérimentales recueillies sur ces deux lots, de 4 vaches chacun, composé Y'un de 2 normandes et de 2 flamandes non castrées et l'autre de 2 normandes et de 2 flamandes castrées après avoir été reconnues réellement comparables aux témoins

En ce qui concerne l'opération elle-même, elle a été pratiquée par M. Lermat de la façon suivante :

La bête étant tenue solidement par la tête, deux aides se placent de chaque côté des hanches pour éviter tout déplacement de l'animal. Après lavage antiseptique et minutieux, extérieur et intérieur, des premières voies génitales, la main de l'opérateur s'introduit dans le vagin qui s'est généralement dilaté sous l'action des injections chaudes. Une ouverture est alors pratiquée à l'aide d'un bistouri à lame cachée, au-dessus du "museau de tanche ", dans les plans charnus et le péritoine. Deux doigts, passant par cette fenêtre, vont à la recherche des ovaires qui, l'un après l'autre,

(1) J. Arqurer : Données expérimentales sur la traite mécanique. Bull. de la Soc. Sc. Hyg. Alim, T. xvu, 1929, no 1, p. 1. 
sont attirés dans la cavité vaginale, puis séparés au moyen d'une chaîne d'écraseur, manœuvrée très lentement. La section des ligaments est faite en deçà de la partie comprimée par la chaîne, ce qui permet, lorsque les vaisseaux sont écrasés incomplètement, d'éviter toute hémorragie au moment où le ligament est abandonné dans la cavité péritonéale. Un dernier lavage interne et externe avec une solution antiseptique termine l'opération.

Nous avons constaté que la castration n'est pas aussi douloureuse qu'on peut se l'imaginer. Les 4 vaches ovariotomisées ne se sont nullement défendues, même les normandes qui étaient des bêtes nerveuses. Il n'a été enregistré aucune élévation de température notable. Les bêtes opérées avaient un appétit normal au repas du soir, le jour même de l'opération; leur lait ne baissa que peu pendant la journée du lendemain et le'surlendemain le rendement avait presque repris son taux initial.

\section{$*^{*} *$}

Afin d'éviter des longueurs de rédaction, toutes les données expérimentales recueillies ont été groupées, tout d'abord, dans des tableaux et graphiques individuels, puis condensées dans les tableaux et graphiques suivants, qui opposent les données moyennes se rapportant, d'une part, aux 4 vaches non castrées servant de témoins, et, d'autre part, aux 4 vaches castrées reconnues rigoureusement comparables aux premières au moment de leur castration.

Pour faciliter la comparaison, ces résultats moyens ont été systématiquement rapportés à une même valeur initiale, par exemple à 500 kilos pour le poids vif d'une vache moyenne, à 10 kilos de lait pour la production par 24 heures et à 100 en ce qui concerne les variations des teneurs du lait en ses principaux composants, telles que trouvées à-1'analyse des échantillons prélevés.

La comparaison des résultats obtenus vise :

d'abord, la production quantitative, soit de lait, soit de viande,

et, ensuite, la qualité de cette production, telle qu'il a paru possible de la mieux définir.

Ces résultats, rappelons-le encore, résument les données obtenues par l'observation des groupes de vaches à comparer pendant 10 à 11 mois consécutifs après le vêlage, soit 3 mois $1 / 2$ à 4 mois avant l'époque de la castration, puis plus de 6 à 7 mois après. Il n'est pas inutile de rappeler que les vaches non castrées n'avaient pas été mises, contrairement à la pratique agricole, en état de gestation. Il s'agissait de comparer les variations de l'activité mammaire au cours d'une lactation complète, sans autre trouble apporté à cette dernière que la castration opérée sur l'un des groupes de vaches à comparer. 
TABLEAU 1 .
Variations des quantités moyennes de lait, en kilos, produites

par 4 vaches castrées et 4 vaches non castrées.

Rapportées à une production de $10 \mathrm{~kg}$. au $1^{\mathrm{er}}$ mars (1 ${ }^{\mathrm{er}}$ contrôle quantitatif après la castration).

\begin{tabular}{|c|c|c|c|c|c|c|c|c|c|c|}
\hline \multirow{2}{*}{$\begin{array}{c}\text { EPOQUES } \\
\text { DES CONTROLKS }\end{array}$} & \multicolumn{4}{|c|}{ NON CASTRÉES } & \multirow{3}{*}{$\begin{array}{l}\text { Moyenne } \\
\text { en kilos }\end{array}$} & \multicolumn{4}{|c|}{ CASTRÉES } & \multirow{3}{*}{$\begin{array}{l}\text { Moyenne } \\
\text { en kilos }\end{array}$} \\
\hline & \multicolumn{2}{|c|}{ Flamandes } & \multicolumn{2}{|c|}{ NORMANDES } & & $\mathrm{FL}$ & DES & Nor & DES & \\
\hline NUMÉROS DES VACHES & 3 & 7 & 9 & 10 & & 4 & 6 & 12 & 15 & \\
\hline 2.4 mars ....... & 0,0 & 10,0 & 10,0 & 10,0 & 10,0 & 10,0 & 10,0 & 10,0 & 10,0 & 10,0 \\
\hline $9.11-$. & 9,6 & 9,0 & 9,6 & 7,8 & 9,0 & 9,9 & 8. & 8,5 & 10,4 & 9,5 \\
\hline$\cdot \cdot \cdot$ & 7,7 & 9,3 & 9,2 & 8,9 & 8,7 & 9,4 & 8,3 & 8 & 10,2 & 8,5 \\
\hline $7-10$ avril . . . . & 7,1 & 8,3 & 8,8 & 7,5 & 7,9 & 8,3 & 8,4 & 7,6 & 9,8 & 8,5 \\
\hline$=\ldots . .$. & 6,6 & 8,1 & 8,6 & 7,5 & 7,7 & 7,6 & 8,5 & 7,3 & 8,7 & 8,0 \\
\hline $20-23-$ & 5,6 & 6,7 & 8,7 & 7,2 & 7,0 & 7,7 & 7,8 & 6,9 & 8,4 & 7,7 \\
\hline 6.7 mai . . . & 5,9 & 8,4 & 9,7 & 7,4 & 7,8 & 7,9 & 8,9 & 7,4 & 9,1 & 8,3 \\
\hline $18-21-\ldots \ldots$ & 5,4 & 8,2 & 8,9 & 7,2 & 7,4 & 7,7 & 8,9 & 7,4 & 9,3 & 8,3 \\
\hline $3 \cdot 4$ juin . . . . & 6,6 & 7,2 & 8,7 & 7,8 & 7,6 & 6,7 & 9,1 & 7,3 & 9,1 & 8,0 \\
\hline $9.10-\ldots . .$. & 5,1 & 7,4 & 8,3 & 6,6 & 6,8 & 7,2 & 8,6 & 7,5 & 9,2 & 8,1 \\
\hline.... & 5,0 & 7,6 & 8,5 & 7,2 & 7,1 & 7,0 & 8,3 & 7,3 & 9,2 & 8,0 \\
\hline$-\ldots . .$. & 5,5 & 6,8 & 8,3 & 6,4 & 6,7 & 7,5 & 8,6 & 6,8 & 9,2 & 8,0 \\
\hline ler juillet. .... & 4,8 & 6,4 & 7,7 & 5,4 & 6,1 & 6,4 & 8,2 & 6,8 & 8,7 & 7,5 \\
\hline $5-6 \quad+\ldots .$. & 4,2 & $5,9^{\circ}$ & 8,2 & 5,8 & 6,0 & 6,3 & 7,7 & 6,0 & 8,4 & 7,1 \\
\hline$=\ldots . .$. & 3,9 & 5,6 & 7,6 & 4,7 & 5,4 & 5,6 & 7,3 & 5,1 & 7,5 & 6,4 \\
\hline....- & 4,0 & 5,5 & 8,0 & 5,0 & 5,6 & 5,5 & 7,2 & 5,5 & 6,7 & 6,2 \\
\hline $29-30-\ldots . .$. & 3,5 & 4,6 & 7,4 & 4,7 & 5,0 & 5,3 & 7,1 & 5,0 & 6,9 & 6,1 \\
\hline 3-6 août . . . . & 3,1 & 4,5 & 6,9 & 5,3 & 5,0 & 4,5 & 6,7 & 5,0 & 6,6 & 5,7 \\
\hline $10.12 \div \ldots . .$. & 3,0 & 4,9 & 7,3 & 5,2 & 5,1 & 5,0 & 6,9 & 5,3 & 6,8 & 6,0 \\
\hline...- & 3,0 & 4,6 & 6,7 & 5,2 & 4,9 & 5,0 & 6,4 & 5,0 & 6,0 & 5,6 \\
\hline $25-28 \quad-\quad .$. & 2,7 & 4,0 & 6,8 & 4,4 & 4,5 & 5,0 & 6,5 & 4,8 & 6,3 & 5,6 \\
\hline 1-4 septembre , . & 2,7 & 4,3 & 6,7 & 4,3 & 4.5 & 4,5 & 6,3 & 4,5 & 6,0 & 5,3 \\
\hline $10-12 \quad-\quad \cdots$ & 2,8 & 4,3 & 7,0 & 4,0 & 4,5 & 4,7 & 6,6 & 4,8 & 6,2 & 5,6 \\
\hline.. & 2,2 & 3,8 & 6,4 & 4,4 & 4,2 & 4,5 & 6,2 & 4., 7 & 6,3 & 5,4 \\
\hline.- .1 & 2,3 & 4,2 & 6,1 & 4,5 & 4,3 & 4,4 & 6,5 & 4,8 & 6,4 & 5,5 \\
\hline
\end{tabular}


INFLUENCE DE LA CASTRATION SUR LA PRODUCTION QUANTITATIVE DE LAIT ET DE VIANDE.

Le tableau I et le graphique I enregistrent les quantités moyennes de lait par tête, produites par les deux groupes de vaches à comparer à partir de l'époque de la castration, étant entendu que, avant l'ovariotomie, le groupe des vaches opérées et le groupe des vaches témoins présentaient, aux divers points de vue intéressants, une véritable similitude. Pour plus de clarté, le tableau et le graphique indiquent les variations de la production individuelle et de la production moyenne, telle qu'elle existait pour les deux groupes à l'époque de la castration ; elle était alors équivalente réellement à $10 \mathrm{~kg}$. de lait par tête et par jour, à quelques centaines de grammes près.

On voit sur le graphique I que, à partir de l'époque de la castration, la production des deux groupes comparables a diminué suivant des rythmes très voisins. A la fin de la lactation cette diminution, par rapport au rendement du début, était :

de $45 \%$ pour les vaches castrées,

de $58 \%$ pour les vaches non castrées,

soit une surproduction minime, en fin de lactation, de $13 \%$ en faveur des vaches castrées.

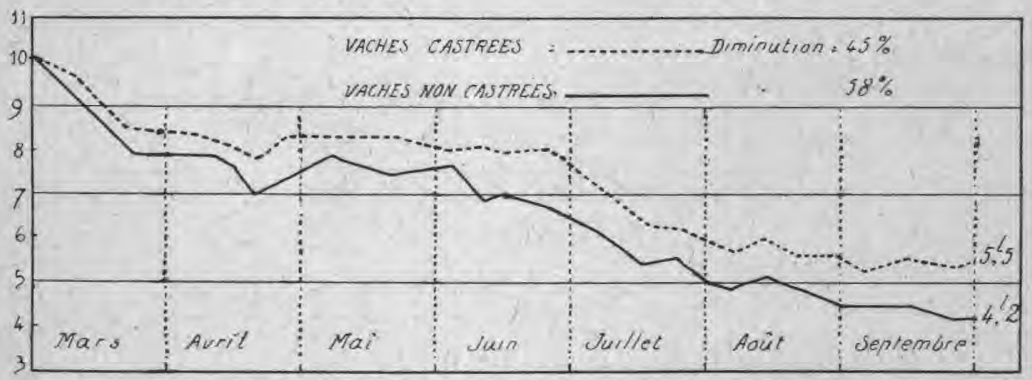

Graphique I

Comparaison des quantités de lait produites par des groupes de vaches comparables, castrées et non eastrées.

Le tableau II suivant permet de se rendre compte de l'influence de la castration comparativement sur les normandes et les flamandes. On enregistre chez les flamandes une action favorable plus accentuée que chez les normandes qui ne réagissent pratiquement pas. Au bout de 7 mois, les flamandes castrées produisaient par tête et par jour $5 \mathrm{~kg}$. 4 de lait et les non castrées $3 \mathrm{~kg}$. 2 pour une production, à l'époque de la castration, de $10 \mathrm{~kg}$.

Le graphique II et le tableau III donnent les variations de poids vif, par tête moyenne, des deux lots à comparer, en les rapportant à un poids par tête de $500 \mathrm{~kg}$. à l'époque de la castration. Il est 
Variations des quantités de lait produites comparativement par des vaches Flamandes, castrées et non castrées, et par des vaches Normandes, castrées et non castrées.

Rapportées à une production de $10 \mathrm{~kg}$. au $1^{\mathrm{er}}$ mars (1 ${ }^{\mathrm{er}}$ contrôle quantitatif après la castration).

\begin{tabular}{|c|c|c|c|c|c|c|c|c|c|c|c|c|}
\hline \multirow{2}{*}{$\begin{array}{c}\text { Epoques } \\
\text { DEs Controles }\end{array}$} & \multicolumn{6}{|c|}{ FLAMANDES } & \multicolumn{6}{|c|}{ NORMANDES } \\
\hline & \multicolumn{3}{|c|}{ CASTRGES } & \multicolumn{3}{|c|}{ NON CASTRÉES } & \multicolumn{3}{|c|}{ CASTREES } & \multicolumn{3}{|c|}{ NON CASTRÉES } \\
\hline Numáros des VACHES & 4 & 6 & Moyenne & 3 & 7 & Moyenne & 12 & 15 & Moyenne & 9 & 10 & Moyenn \\
\hline 2.4 mars . . . . & 10,0 & 10,0 & 10,0 & 10,0 & 10,0 & 10,0 & 10,0 & 10,0 & 1,00 & 10,0 & 10,0 & 10,0 \\
\hline$. \quad . \quad$ & 9,9 & 8 & 8,9 & 9,6 & 9,0 & 9,3 & 8,5 & 10,4 & 9,4 & 9,6 & 7,8 & 8,7 \\
\hline - . . . . & 9,4 & 8,3 & 8,8 & 7,7 & 9,3 & 8,5 & 8,0 & 10,2 & 9,1 & 9,2 & 8,9 & 9,0 \\
\hline $7-10$ avril & 8,3 & 8,4 & 8,3 & 7,1 & 8,3 & 7,7 & 7,6 & 9,8 & $8,7^{*}$ & 8,8 & 7,5 & 8,1 \\
\hline - . . . . & 7,6 & 8,5 & 8,0 & 6,6 & 8,1 & 7,3 & $.7,3$ & 8,7 & 8,2 & 8,6 & 7,5 & 8,0 \\
\hline $20.23=-\ldots .$. & 7,7 & 7,8 & 7,7 & 5,6 & 6,7 & 6,1 & 6,9 & 8,4 & 7,6 & 8,7 & 7,2 & 7,9 \\
\hline 6.7 mai . . . & 7,9 & 8,9 & 8,4 & 5,9 & 8,4 & 7,1 & 7,4 & 9,1 & 8,2 & 9,7 & 7,4 & 8,5 \\
\hline $18-21-\ldots$ & 7,7 & 8,9 & 8,3 & 5,4 & 8,2 & 6,8 & 7,4 & 9,3 & 8,3 & 8,9 & 7,2 & 8,0 \\
\hline $3-4$ juin ..... & 6,7 & 9,1 & 7,9 & 6,6 & 7,2 & 6,9 & 7,3 & 9,1 & 8,2 & 8,7 & 7,8 & 8,2 \\
\hline $9.10-\ldots .$, & 7,2 & 8,6 & 7,9 & 5,1 & 7,4 & 6,2 & 7,5 & 9,2 & 8,3 & 8,3 & 6,6 & 7,4 \\
\hline $15-16-\because \cdots$. & 7,0 & 8,3 & 7,6 & 5,0 & 7,6 & 6,3 & 7,3 & 9,2 & 8,2 & 8,5 & 7,2 & 7,8 \\
\hline $22.25-\ldots . .$. & 7,5 & 8,6 & 8,0 & 5,5 & 6,8 & 6,1 & 6,8 & 9,2 & 8,0 & 8,3 & 6,4 & 7,3 \\
\hline Ier juillet. . & 6,4 & 8,2 & 7,3 & 4,8 & 6,4 & 5,6 & 6,8 & 8,7 & 7.7 & 7,7 & 5,4 & 6,5 \\
\hline - . . . & 6,3 & 7,7 & 7,0 & 4,2 & 5,9 & 5,0 & 6,0 & 8,4 & 7,2 & 8,2 & 5,8 & 7,0 \\
\hline $16.17 \quad-\ldots$. & 5,6 & 7,3 & 6,4 & 3,9 & 5,6 & 4,7 & 5,1 & 7,5 & 6,3 & 7,6 & 4,7 & 6,1 \\
\hline $22.23=-\ldots$ & 5,5 & 7,2 & 6,3 & 4,0 & 5,5 & 4,7 & 5,5 & 6,7 & 6,1 & 8,0 & 5,0 & 6,5 \\
\hline $29.30-. .$. & 5,3 & 7,1 & 6,2 & 3,5 & 4,6 & 4,0 & 5,0 & 6,9 & 5,9 & 7,4 & 4,7 & 6,0 \\
\hline 3-6 abût . . . & 4,5 & 6,7 & 5,6 & 3,1 & 4,5 & 3,8 & 5,0 & 6,6 & 5,8 & 6,9 & 5,3 & 6,1 \\
\hline $10.12-$ & 5,0 & 6,9 & 6,4 & 3,0 & 4,9 & 3,9 & 5,3 & 6,8 & 6,0 & 7,3 & 5,2 & 6,2 \\
\hline $17.20-$ & 5,0 & 6,4 & 5,7 & 3,0 & 4,6 & 3,8 & 5,0 & 6,0 & 5,5 & 6,7 & 5,2 & 5,9 \\
\hline $25-28-\ldots$. & 5,0 & 6,5 & 5,7 & 2,7 & 4,0 & 3,3 & 4,8 & 6,3 & 5,5 & 6,8 & 4,4 & 5,6 \\
\hline 14 septembre. . & 4,5 & 6,3 & 5,4 & 2,7 & 4,3 & 3,5 & 4,5 & 6,0 & 5,2 & 6,7 & 4,3 & 5,5 \\
\hline $10.12 \quad-$. & 4,7 & 6,6 & 5,6 & 2,8 & 4,3 & 3,5 & 4,8 & 6,0 & 5,4 & 7,0 & 4,0 & 5,5 \\
\hline $24 \cdot 25$ & 4,5 & 6,2 & 5,3 & 2,2 & 3,8 & 3,0 & 4,7 & 6,3 & 5,5 & 6,4 & 4,4 ;A & 5,4 \\
\hline $27-28-$ & 4,4 & 6,5 & 5,4 & 2,3 & 4,2 & 3,2 & 4,8 & 6,4 & 5,6 & 6,1 & 4,5 & 5,3 \\
\hline
\end{tabular}


TABLEAU III.

Variations du poids vif moyen, par tête, à partir de l'époque de la castration de 4 vaches castrées et de 4 vaches non castrées.

Rapportées au poids par tête de $500 \mathrm{~kg}$. à l'époque de la castration.

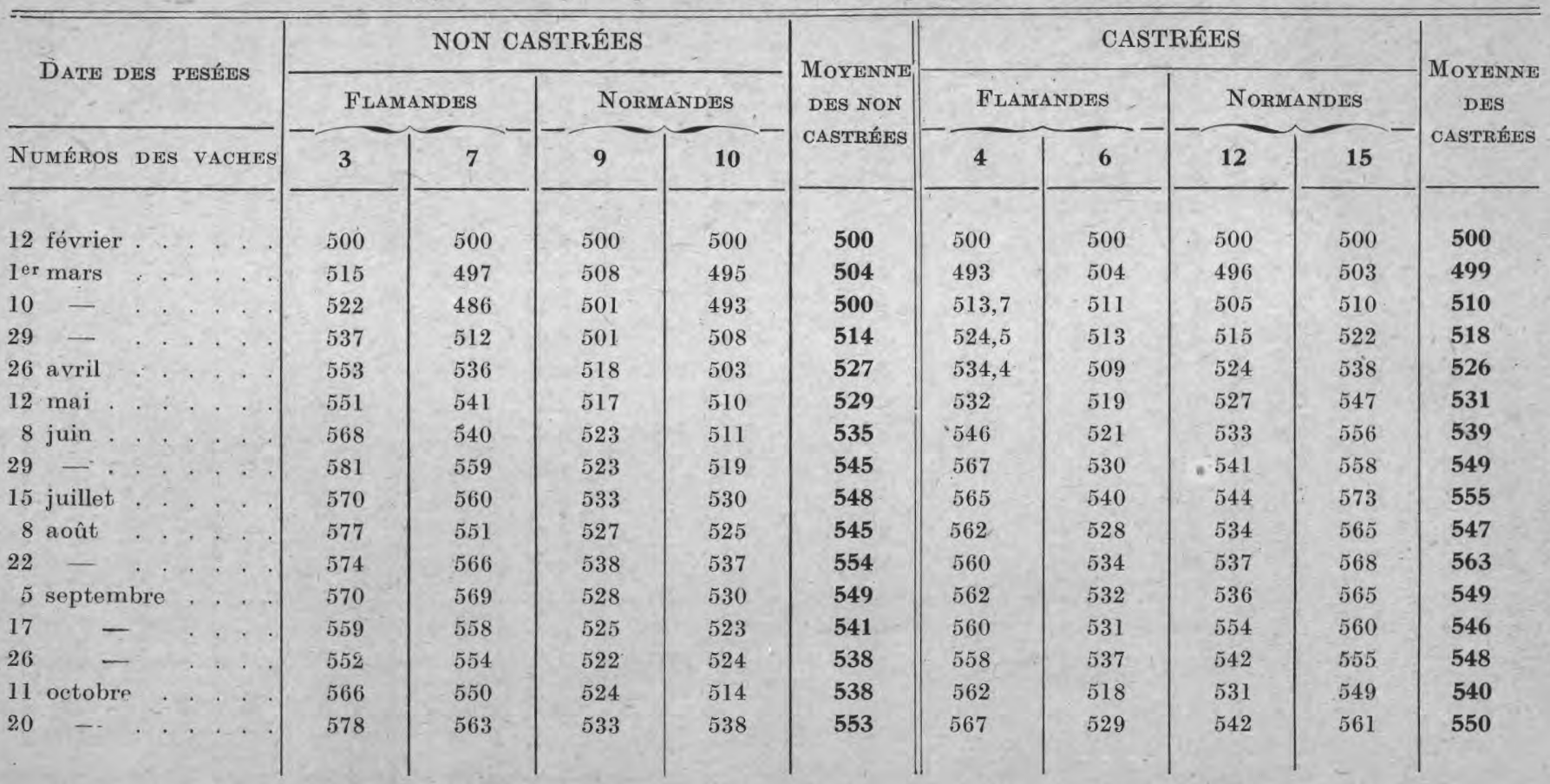


net que la castration n'a pas favorisé l'engraissement, alors que l'alimentation était rigoureusement la même, tant au point de vue quantitatif que qualitatif.

Si l'on tient compte des différences de race des vaches mises comparativement en observation et nourries exactement de même, ainsi que l'on peut le constater sur le tableau IV, les flamandes castrées ont moins augmenté de poids que les non castrées, $548 \mathrm{~kg}$. au lieu de $570 \mathrm{~kg}$., soit un meilleur rendement de viande, pour ces dernières, de $22 \mathrm{~kg}$. en 8 mois. Par contre, le croît, pendant la même période, des normandes castrées, $51 \mathrm{~kg}$., s'est trouvé supérieur à celui des témoins non castrées, $35 \mathrm{~kg}$.

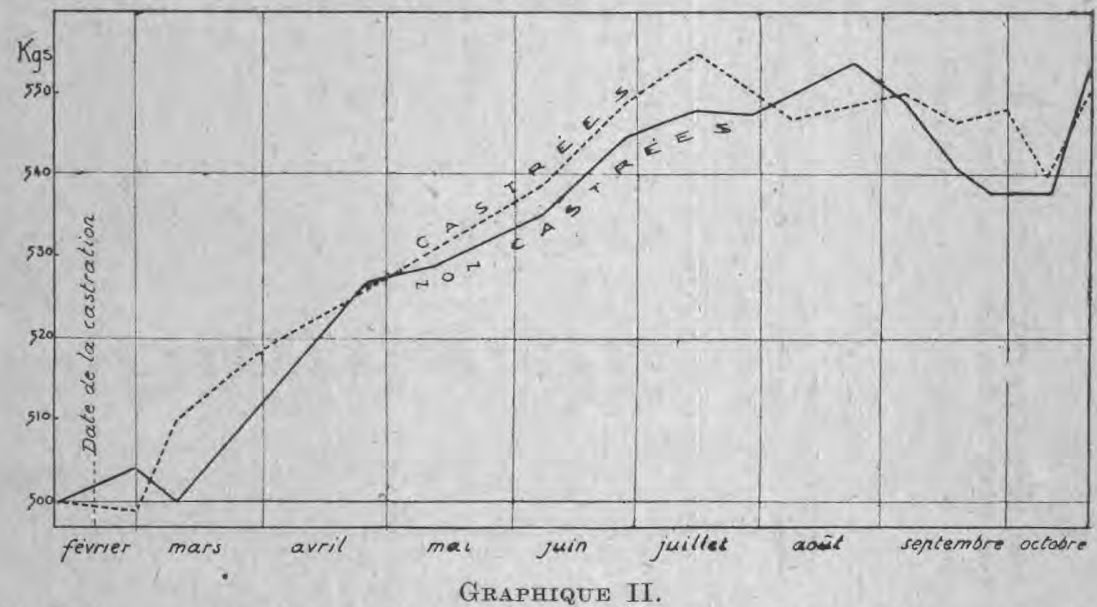

Variations comparatives du poids vif de deux groupes de vaches comparables castrées et non castrées.

En ce qui concerne l'effet de la castration sur la production quantitative du lait, il est intéressant de signaler que les changements d'alimentation ont une action rigoureusement parallèle chez les vaches castrées et non castrées.

Le graphique III et le tableau VI montrent comment ont agi d'abord la substitution de la ration $B$, sans tourteaux mais plus riche en sons, à la ration $\mathrm{A}$ avec tourteaux, puis le retour à la ration $\mathrm{A}$. Les formules et caractéristiques, rappelées dans le tableau $V$, de ces rations, de valeurs calorifiques très voisines, ne diffèrent, à proprement parler, du point de vue de la composition chimique, que par une teneur plus faible de la ration $B$, en matières azotées et également en matières minérales, mais plus élevée en glucides (sucres, amidon, pentosanes, etc...). C'est là un exemple typique des conséquences possibles, souvent méconnues, d'un simple changement d'équilibre alimentaire (1).

(1) J, Alquier : Contribution à l'étude des équilibres alimentaires. Ce Bulletin, T. XIV, 1926, n० 10, pp. 496 et suiv. 
TABLEAU IV.

Variations du poids vif moyen par tête, à partir de l'époque de la castration chez des Flamandes castrées et non castrées et des Normandes castrées et non castrées.

Rapportées au poids par tête de $500 \mathrm{~kg}$. à l'époque de la castration.

\begin{tabular}{|c|c|c|c|c|c|c|c|c|c|c|c|c|}
\hline \multirow{3}{*}{$\begin{array}{c}\text { DATES } \\
\text { DES CONTROLES } \\
\text { NUMÉros DES VACHES }\end{array}$} & \multicolumn{6}{|c|}{ FLAMANDES } & \multicolumn{6}{|c|}{ NORMANDES } \\
\hline & \multicolumn{3}{|c|}{ CASTRÉES } & \multicolumn{3}{|c|}{ NON CASTREES } & \multicolumn{3}{|c|}{ CASTRRÉES } & \multicolumn{3}{|c|}{ NON CASTRÉES } \\
\hline & 4 & 6 & Moyenne & 3 & 7 & Moyenne & -12 & 15 & Moyenne & 9 & 10 & Moyenne \\
\hline 12 février . . . . & 500 & 500 & 500 & 500 & 500 & 500 & 500 & 500 & 500 & 500 & -500 & 500 \\
\hline...$\quad$. & 493 & 504 & 498 & 515 & 497 & 506 & 496 & 503 & 500 & 508 & 495 & 501 \\
\hline$\therefore .$. & 513,7 & 511 & 512 & 522 & 486 & 504 & 505 & 510 & 507 & 501 & 493 & 497 \\
\hline$. \quad . \quad . \quad$. & 524,5 & 513 & 518 & 537 & 512 & 524 & 515 & 522 & 518 & 501 & 508 & 504 \\
\hline 26 avril & 534,4 & 509 & 521 & 553 & 536 & 544 & 524 & 538 & 531 & 518 & 503 & 510 \\
\hline ...... & 532 & 519 & 525 & 551 & 541 & 546 & 527 & 547 & 537 & 517 & 500 & 508 \\
\hline 8 juin . . . . . & 546 & 521 & 533 & 568 & 540 & 554 & 534 & 566 & 550 & 523 & 511 & 517 \\
\hline . . . . . & 567 & 530 & 548 & 581 & 559 & 570 & 542 & 558 & 550 & 523 & 519 & 521 \\
\hline 15 juillet. . . . . & 565 & 540 & 552 & 570 & 560 & 565 & 544 & 573 & 575 & 533 & 530 & 531 \\
\hline 8 août. . . . . & 562 & 528 & 545 & 577 & 5.51 & 564 & 534,5 & 565 & 550 & 527 & 525 & 526 \\
\hline $22 \div \ldots . \ldots$ & 560 & 534 & 547 & 574 & 566 & 570 & 537 & 568 & 552 & 538 & 537 & 537 \\
\hline 5 septembre & 562 & 532 & 547 & 570 & 569 & 569 & 536 & 565 & 550 & 528 & 530 & 529 \\
\hline $17-$ & 560 & 531 & 545 & 559 & 558 & 558 & 534 & 560 & 547 & 525 & -523 & 524 \\
\hline $26 \quad-\quad$. & 558 & 537 & 547 & 552 & 554 & 553 & 542 & 555 & 548 & 522 & 524 & 523 \\
\hline 11 octobre. . . . & 562 & 518 & 540 & 566 & 550 & 558 & 531 & 549 & 540 & 524 & 514 & 519 \\
\hline $20-\quad . .$. & 567 & 529 & 548 & 578 & 563 & 570 & 542 & 561 & 551 & 533 & 538 & 535 \\
\hline
\end{tabular}




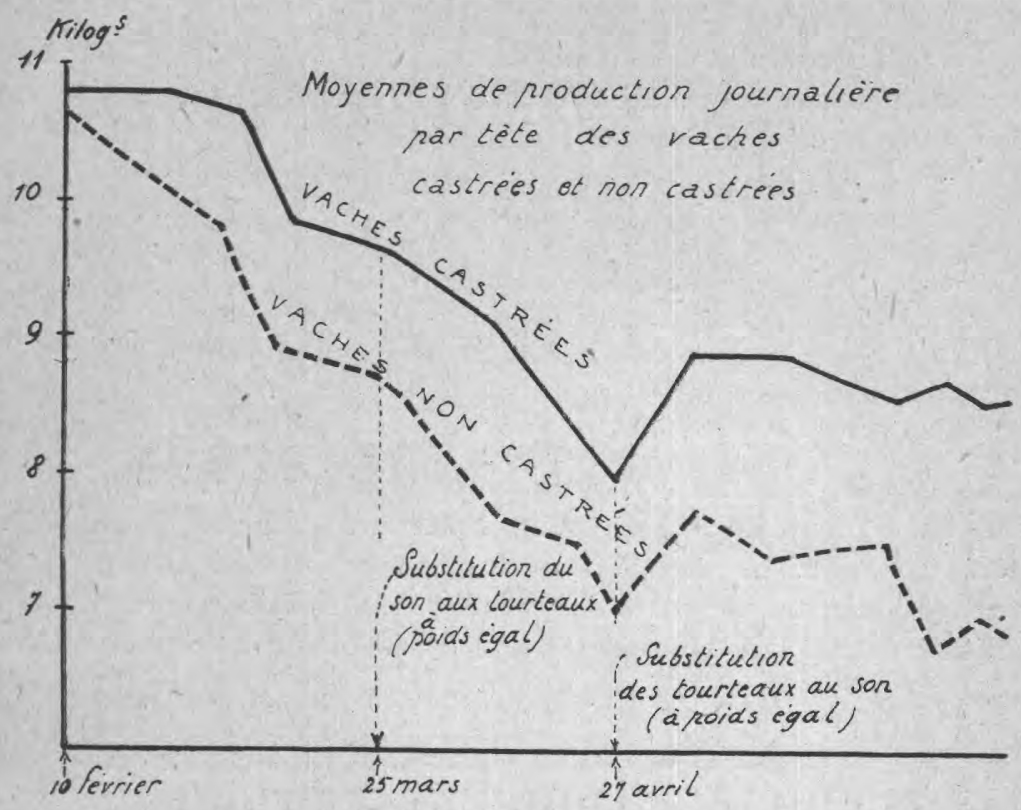

Graphique III.

Influence de différents changements d'alimentation sur la production du lait par des vaches castrées et non castrées.

TABLEAU $V$.

\section{Formules}

Foin.

Paille mélassée

Paille hachée

Sons .

Mouture de manioc

Farine de tourteau d'arachide.

Farine de tourteau de lin.

Composition chimique de la matière sèche Matières minérales

Matières grasses

Matières azotées

Sucres préexistant

Amidon digestible

Pentosanes

Cellulose pure

Indéterminés et Lignines

Valeur ealorifique.

\begin{tabular}{|c|c|}
\hline $\begin{array}{c}\text { Ration } A \\
\text { avec tourteaux } \\
\text { 10 jusquau26 mars } \\
2^{\circ} \text { depuisle } 27 \text { avril }\end{array}$ & $\begin{array}{c}\text { Ration B } \\
\text { sans tourteaux } \\
\text { du } 26 \text { mars } \\
\text { au } 27 \text { avril }\end{array}$ \\
\hline kg. & $\mathrm{kg}$. \\
\hline 5,0 & 5,0 \\
\hline 2,0 & 2,0 \\
\hline 1,0 & 1,0 \\
\hline 2,5 & 3,7 \\
\hline 2,5 & 2,5 \\
\hline 0,6 & n) \\
\hline 0,6 & ¿ \\
\hline Gr. & Gr. \\
\hline 870 & $852=$ \\
\hline 209 & 208 \\
\hline 1.366 & 1.140 \\
\hline 941 & 961 \\
\hline 2.650 & 2.784 \\
\hline 1.939 & 2.141 \\
\hline 949 & 964 \\
\hline 4.189 & 4.120 \\
\hline $47.099 \mathrm{cal}$. & 48.275 cal. \\
\hline
\end{tabular}


Sous l'influence de ees trois changements successifs de ration, la quantité totale du lait produite en 24 heures par les vaches soumises au contrôle a varié de la façon suivante :

TABLEAU VI.

Lait total produit

Ration A avec tourteaux, donnée jusqu'au 16 mars .

$143 \mathrm{~kg} ., 7$

Suppression progressive des tourteaux du 16 au 26 mars .

$136 \mathrm{~kg}, 5$

Ration B sans tourteaux, donnée du 26 mars au 27 avril .

$122 \mathrm{~kg} ., 3$

Retour progressif à la Ration $\mathrm{A}$ avec tourteaux, à partir du ler mai.

$137 \mathrm{~kg} ., 1$

Le graphique III montre bien que les variations du régime alimentaire ont influencé de même toutes les vaches, qu'elles soient castrées ou non. Le parallélisme des productions est ici rigoureux. Nous reviendrons, dans la suite, sur les modifications biologiques et qualitatives du lait résultant des mêmes changements de ration.

(A suivre.)

\section{BIBLIOGRAPHIE ANALYTIQUE}

\section{LES LIVRES}

Burke (A. D.). - Practical Dairy Test (Essais pratiques de la laiterie. Un manuel pour les étudiants et ceux qui sont intéressés aux essais pratiques des produits laitiers). The Olsen Publishing Co., Ed., Milwaukee, Wisconsin. Un vol. relié toile de $206 \mathrm{pp}$.

Comme le dit l'auteur dans la courte préface qu'il donne en première page, son manuel n'est pas une publication chimique. Il a été écrit dans le but de servir, non seulement aux étudiants de la laiterie, mais aussi aux travailleurs des usines laitières.

Le texte est accompagné d'images représentant tous les appareils dont on peut se servir, ce qui a pour but de rendre plus claires les explications du texte. Toute formule complexe est évitée. La façon dont une épreuve doit être conduite est décrite avec le plus grand soin, ce qui se comprend étant donné que le lecteur auquel cet ouvrage est destiné n'a pas toujours une très grande instruction.

Ch. Porcherer.

Sammis (J. L.), - Cheese Making (Fabrication du fromage. Un livre pour les praticiens de la fromagerie, les Ecoles d'agriculture et de laiterie). Un vol. relié toile de 295 pp., illustré, édité par The Cheese Maker Book Co., Madison, Wisconsin, U.S.A., 1930.

La production du fromage et sa consommation ont considérablement augmenté aux Etats-Unis dans ces dernières années.

Il n'est pas douteux que le régime " sec " ainsi que l'heureuse campagne faite en faveur de la consommation du lait et de ses dérivés aux Etats-Unis ne soit pour 